\title{
Higher adherence to the Mediterranean Diet is associated with weight loss maintenance: the MedWeight study
}

\section{Abstract}

Introduction: Adherence to the Mediterranean dietary pattern has been associated with numerous health benefits in noncommunicable diseases, including obesity management. However, the associations of the Mediterranean Diet with weight loss maintenance remain to be evaluated.

Methods: We analyzed data from 500 participants (61\% women) of the MedWeight study. Eligible volunteers were men and women 18-65 years old, reporting an intentional weight loss of at least $10 \%$, starting from a BMI $\geq 25 \mathrm{~kg} / \mathrm{m}^{2}$. Based on their current weight, participants were characterized as maintainers (current weight $\leq 90 \%$ of maximum weight), or regainers (current weight $>95 \%$ of maximum weight). Socio-demographics, lifestyle measurements and weight history were recorded. Dietary intake was assessed by 2 telephone 24-hour recalls. Adherence to the Mediterranean Diet was assessed with the MedDietScore (range 0-55, greater scores showing higher adherence). Physical activity levels were assessed with the International Physical Activity Questionnaire-short form. Results are expressed as means \pm SD, frequencies $(\%)$ or Odds Ratio [OR; $95 \%$ Confidence Interval].

Results: Compared to regainers $(31 \%)$, maintainers were younger $(31.4 \pm 10.0$ vs. $36.6 \pm 10.8$ years, $p<0.001)$, had lower BMI $(25.7 \pm$ 4.3 vs. $\left.31.4 \pm 5.1 \mathrm{~kg} / \mathrm{m}^{2}, \mathrm{p}<0.001\right)$, and had greater initial body weight loss $(25.5 \pm 8.6 \%$ vs. $18.4 \pm 6.9 \%, \mathrm{p}<0.001)$. Sex and years of formal education were not significantly different between maintainers and regainers $(p>0.05)$. Being in the highest MedDietScore quintile (vs. the lowest) was associated with $91 \%$ higher odds of being a maintainer [crude OR $=1.91 ; 1.05-3.45$ ]. This association remained significant after adjusting for sex, age, physical activity level (METŸminutes/week) and energy intake (kcal/day) [adjusted $\mathrm{OR}=2.01 ; 1.05-3.83]$.

Discussion: Higher adherence to the Mediterranean Diet was independently associated with 2-fold increased likelihood of weight loss maintenance. Our results highlight the favorable effects of a prudent dietary pattern in long-term obesity management, as well as novel targets for diet planning during weight loss maintenance.

\section{Conflict of Interest}

There is no conflict of interest 